\section{Frequência e fatores de risco da obesidade em uma população de gatos domésticos no Rio de Janeiro}

\author{
Frequency and risk factors of obesity in a population of \\ domestic cats from Rio de Janeiro
}

\author{
Raquel Sampaio Alves', Renée Cristine Carvalho Barbosa²*, Margarete Weinschutz Gheren', \\ Licínio Esmeraldo da Silva ${ }^{3} \&$ Heloisa Justen Moreira de Souza ${ }^{4}$ \\ 'Médicas veterinárias, MScs. Programa de Pós-graduação em Medicina Veterinária, Universidade Federal Rural do Rio de \\ Janeiro - UFRRJ, Seropédica, RJ, Brasil \\ 2Médica veterinária, MSc. Programa de Pós-graduação em Clinica e Reprodução Animal, Universidade Federal Fluminense - \\ UFF, Niterói, RJ, Brasil \\ ${ }^{3}$ Matemático, Dr. Departamento de Estatística, Instituto de Matemática, Universidade Federal Fluminense - UFF, \\ Niterói, RJ, Brasil \\ ${ }^{4}$ Médica veterinária, Dra. Departamento de Medicina e Cirurgia Veterinária, Instituto de Veterinária, Universidade Federal \\ Rural do Rio de Janeiro - UFRRJ, Seropédica, RJ, Brasil
}

\section{Resumo}

A obesidadeé uma condição de balanço energético positivo e excessiva formação de tecido adiposo com efeitos diretos na morbidade e mortalidade. O objeitvo deste trabalho foi investigar a condição corporal de uma população de gatos, na "realidade brasileira", identificando a freqüência de sobrepeso e obesidade, apontando os fatores de risco encontrados para esta amostra e demonstrar a percepção do proprietário sobre a condição corporal do seu gato. Este é o primeiro estudo no país relacionado à epidemiologia da condição da obesidade somente na espécie felina. Para isto, foram selecionados 106 animais da espécie felina (Felis catus), encaminhados para realização algum procedimento cirúrgico, machos e fêmeas inteiros ou esterilizados, com ou sem raça definida. Estes animais foram atendidos em uma clínica privada exclusiva no atendimento de gatos no Rio de Janeiro, no período de junho a novembro de 2010. Os gatos foram avaliados por meio de um questionário respondido por seus donos a cerca da rotina do gato e informações demográficas sobre o proprietário, além de uma avaliação subjetiva e uma objetiva para a obesidade. Neste estudo a análise subjetiva foi feita por meio do escore de cinco pontos e a objetiva pelo cálculo do índice de massa corporal do felino (IMCF). Doenças relacionadas à obesidade foram também observadas e descritas. As idades dos gatos e dos proprietários e peso dos gatos foram avaliados através das suas médias e seus respectivos desvios padrão. Os diversos fatores de risco presumidos foram avaliados por meio da razão de chances e os respectivos intervalos de confianças. Conclui-se que a freqüência de excesso de peso na população de gatos da rotina cirúrgica foi de 60,4\%, sendo $23,6 \%$ identificados com sobrepeso e 36,8\% obeso através da avaliação do escore corporal. A obesidade foi evidenciada em metade desta população estudada pelo método do índice de massa corporal do felino. Os fatores de risco para obesidade foram gatos de meia idade, castrados, especialmente machos domiciliados com acesso livre ao alimento. A subestimação do escore por seus proprietários também foi um fator predisponente para obesidade felina. Além disso, a associação do método subjetivo (ECC) com o objetivo (IMCF) foram formas determinantes e concisas para uma adequada avaliação da obesidade.

Palavras-chave: obesidade, felino, escala de condição corporal, índice de massa corporal, fatores de risco.
Como citar: Alves, R. S., Barbosa, R. C. C., Gheren, M. W., Silva, L. E., \& Souza, H. J. M. (2017) Frequência e fatores de risco da obesidade em uma população de gatos domésticos no Rio de Janeiro. Brazilian Journal of Veterinary Medicine. 39(1), 33-45. doi: 10.29374/2527-2179.bjvm0081

Fonte de financiamento: Nenhuma.

Conflito de interesses: Os autores declaram não haver conflito de interesses que precisam ser informados

Recebido: Julho 29, 2017.

Aceito: Novembro 07, 2017

O estudo foi realizado na Universidade Federal Rural do Rio de Janeiro - UFRRJ, Seropédica, RJ, Brasil.

\section{*Correspondência}

Renée Cristine Carvalho Barbosa

Programa de Pós-graduação em Clinica e Reprodução Animal, Universidade Federal Fluminense - UFF

Rua 21 de abril, 14, Bairro Paraíso

CEP 26297-186 - Nova Iguaçu (RJ), Brasil E-mail: reneecristine@veterinaria.med.br

\begin{abstract}
Obesity is a condition of positive energy balance and excessive adipose tissue formation with direct effects on morbidity and mortality. The aim of this study was to investigate body condition in a population of cats within the "Brazilian reality", identifying the prevalence of overweight and obesity, pointing out the risk factors found for this sample and to demonstrate the owner's perception about the condition of his cat body. This is the first study in the country related to the epidemiology of the condition of obesity only in felines. For this, we selected 106 animals of the feline species (Felis catus), referred for a surgical procedure, males and females or sterilized whole, with or mongrel. These animals were treated in a private practice in caring for cats exclusive, located on Rio de Janeiro, from June to November 2010. The cats were evaluated by means of a questionnaire answered by their owners about its routine and demographic information about the owner, besides a subjective and an objective assessment for obesity. In this study
\end{abstract}

Copyright Alves et al. Este é um artigo publicado em acesso aberto (Open Access) sob a licença Creative Commons Attribution Non-Commercial, que permite uso, distribuição e reprodução em qualque meio, sem restrições desde que sem fins comerciais e que o trabalho original seja corretamente citado. 
the subjective analysis was performed using five-point score and objectively by calculating the body mass index of the cat (IMCF). Diseases related to obesity were also observed and described. The ages of cats and cat owners and weight were evaluated by their means and their standard deviations. The various risk factors assumed were evaluated by odd ratios and their confidence intervals. It was concluded that the frequency of overweight in the population of cats from a routine basis was $60.4 \%$, of which $23.6 \%$ identified with overweight and obese $36.8 \%$ through the evaluation of body condition. Obesity was found in half of the population studied by the method of body mass index of the cat. Risk factors for obesity were middle-aged cats, neutered, especially males domiciled with free access to food. The underestimation of the score by their owners was also a predisposing factor for feline obesity. In addition, associating the subjective method (BCE) with the objective (IMCF) were decisive and concise forms for an adequate assessment of obesity.

Keywords: obesity, feline, body condition score, body mass index, risk factors.

\section{Introdução}

A obesidade é uma condição corporal comum encontrada atualmente em medicina interna de pequenos animais. Estima-se que 20 a 40\% dos gatos domésticos norte-americanos sejam obesos (Crystal, 2009). Alguns autores já consideram a obesidade como o distúrbio nutricional mais comum em cães e gatos nos países ocidentais (Zoran, 2010). Há muitos fatores, tanto ambientais como genéticos, que influenciam direta ou indiretamente o equilíbrio energético (Zoran, 2010).

No Brasil, até então, não existiam dados epidemiológicos que refletissem a realidade de gatos com excesso de peso, mesmo não sendo raro identificá-los em nossa na rotina clínica e cirúrgica.

O tecido adiposo, antes conhecido como um "depósito de combustível passivo", hoje é reconhecido como um órgão endócrino ativo que se comunica com o cérebro e os tecidos periféricos por meio da secreção de uma ampla gama de hormônios e fatores de proteína, coletivamente denominados adipocinas (German \& Martin, 2008). A importância do estudo da obesidade decorre de seu papel na fisiopatologia de diversas doenças e da sua capacidade de exacerbar doenças pré-existentes. Em gatos obesos há um aumento da incidência de diabete melito, osteoartrite, dermatite, doença do trato urinário inferior, constipação, lipidose hepática, problemas cardiorrespiratórios (Colliard et al., 2009) e até mesmo neoplasias (Lund et al., 2005; Lafflame, 2006).

O reconhecimento dessa condição de obesidade é o passo inicial para diagnostica-la. Existem inúmeros métodos para avaliar a obesidade, como pesagem, graduação do escore corporal, a mensuração zoométrica ou índice de massa corporal do felino (IMCF), a mensuração de hormônios e a mensuração da gordura pela técnica de absortometria radiológica com feixes duplos (DEXA), entre outros (German, 2006). A classificação do grau de obesidade por meio de escore corporal e o índice de massa corporal são técnicas relativamente simples e requerem habilidade e prática do examinador.

Gatos de meia idade, castrados, sem raça definida, compõem o principal grupo para obesidade felina (Kienzle \& Bergler, 2006; Scarlett et al., 1994). Inatividade, restrição de espaço e de exercício (Robertson, 1999), alimentação ad libitum (Kienzle \& Bergler, 2006) e a subestimação da condição corporal do gato por seus proprietários são fatores que contribuem com a obesidade felina (Allan et al., 2000; Kienzle \& Bergler, 2006).

Novos estudos abrem campos de pesquisas para o diagnóstico, controle da obesidade e da nutrição, portanto, a identificação precisa da obesidade e a investigação dos fatores predisponentes são de grande relevância na qualidade de vida da população felina.

Este estudo tem por objetivo investigar a condição corporal em uma população de gatos, identificando a freqüência de sobrepeso e obesidade na população estudada. Avaliar os fatores de risco associados ao excesso de peso corporal e analisar a percepção dos proprietários quanto à condição corporal do seu gato.

\section{Material e métodos}

O projeto foi realizado em clínica veterinária particular, exclusiva no atendimento de gatos, localizada no bairro de Botafogo, na cidade do Rio de Janeiro, onde foram selecionados, no período de junho a novembro de 2010, 106 animais da espécie felina (Felis catus), machos e 
fêmeas inteiros ou esterilizados, com ou sem raça definida, escolhidos aleatoriamente entre os gatos encaminhados para intervenções cirúrgicas e autorizados por seus proprietários.

Aos proprietários foi solicitado que respondessem um questionário que constava de perguntas com respostas em múltipla escolha, enquanto aguardavam o procedimento. Uma estimativa do peso dos felinos foi requerida, além da satisfação quanto à aparência corporal de seu animal. Este julgamento foi realizado por meio da marcação da silhueta felina que mais se aproximava com a do gato, mediante a apresentação de um diagrama com cinco silhuetas, com figuras correspondentes ao escore visual de 5 pontos, dispostas de forma aleatória.

Buscou-se, ainda, determinar os possíveis fatores de risco relacionados à obesidade felina investigando os hábitos alimentares dos animais, verificando o tipo alimentação principal oferecida, a pessoa responsável pela alimentação do gato, o acesso ao alimento, a freqüência com que era oferecido o alimento e quem determinava o tipo de alimentação. A exposição a outros alimentos diferentes da alimentação principal foi inquirida e a freqüência com que era dado tal alimento. Além desses dados, a atividade física dos gatos também foi investigada. Se o animal era ativo ou não e, em caso de resposta afirmativa, que tipo de atividade realizava.

Igualmente, informações relacionadas ao perfil dos proprietários foram questionadas para o reconhecimento de fatores provavelmente envolvidos na obesidade felina, tais como: idade, sexo, bairro onde mora, o tipo de moradia, estado civil e o número de pessoas morando na casa. Todos os proprietários estavam cientes sobre o estudo e que as informações seriam utilizadas somente para fins relacionados a esta pesquisa.

Todos os gatos incluídos neste trabalho tiveram os seguintes itens analisados: peso corporal (Kg), escore de condição corporal (ECC) e o índice de massa corporal do felino (IMCF). O registro do peso foi feito sempre na mesma balança. Logo após, foi realizada a avaliação subjetiva seguindo protocolo da escala adaptada por Colliard et al. (2009) ciitado por Lafflame (1997). Em seguida, realizada a avaliação da objetiva da obesidade (morfométrica) segundo Butterwick \& Marckwell (1996) pelo mesmo profissional durante todo o estudo. A existência de doenças previamente conhecidas sobre cada animal e relacionadas à obesidade também foi indagada, como, também, se o animal estava sob uso de algum tratamento e/ou medicamento. Quando uma ou mais doenças ligadas à obesidade foram identificadas, estas foram reunidas na ficha do respectivo paciente.

Os dados colhidos foram compilados no programa Excel 2007 (Microsoft $\left.{ }^{\circledR}\right)$ e as análises estatísticas foram processadas no Instituto de Matemática no Departamento de Estatística da Universidade Federal Fluminense (UFF) através do programa estatístico SPSS versão 10.0 para Windows 9.1 (SPSS ${ }^{\circledR}$, IBM Company ${ }^{\circledR}$, NC, EUA).

As idades dos gatos e dos proprietários e peso dos gatos foram avaliados através das suas médias e seus respectivos desvios padrão. A concordância entre a estimativa do ECC do proprietário e do veterinário foi medido pelo coeficiente kappa $(\kappa)$ de Cohen no qual 0,61 $\leq 0 \leq 1$ : alta concordância; 0,41 $\leq \mathrm{k} \leq 0,75$ : concordância moderada; $\mathrm{O} \leq \kappa<0,40$ concordância fraca (todos os intervalos considerados além da concordância casual).

Para o peso corporal (estimado e real), o nível de concordância foi medido por um coeficiente de correlação de Pearson (r). A associação linear entre variáveis contínuas foi investigada por este coeficiente. O modelo linear de associação entre variáveis contínuas foi construído por meio de uma reta de regressão linear, cuja aderência dos dados ao modelo foi avaliada pelo coeficiente de determinação (r2) (o qual varia de $\mathrm{O}$ a 1 - ou de $\mathrm{O}$ a 100 se expresso em percentuais - indicando quanto mais próximo de 1, mais o modelo explica o relacionamento linear entre os dados).

A associação entre os escores de condição corporal e os vários fatores de risco presumidos e o índice de massa corporal felina com os vários fatores de risco presumidos foram avaliados por meio da razão de chances (odd rates) e os respectivos intervalos de confianças a 95\% (IC 95\%). Quando o IC 95\% não incluir o valor 1,00 a associação foi declarada estatisticamente significativa. Valores das razões de chances e intervalos de confianças menores que 1,00 indicaram fatores de proteção para obesidade e valores superiores a 1,00 indicaram fatores de risco (Colliard et al., 2009).

Para as doenças relacionadas à obesidade os gatos foram divididos em dois grupos (obesos e não-obesos) e intervalos de confianças também foram feitos para cada doença relacionada dentro de cada grupo. 


\section{Resultados}

\section{Perfil dos animais}

A média de idade encontrada dos gatos foi de $5,4( \pm 4,39)$ anos. A menor idade foi de cinco meses e a maior, de 18 anos. Um total de 36 (34,0\%) dos gatos estavam situados na faixa etária entre um e dois anos de idade. Já na faixa abaixo de um ano de idade houve apenas 7 (6,6\%) animais. Foram encontrados 10 (9,4\%) dos animais na faixa de dois até cinco anos. Observou-se que na faixa de cinco até nove anos encontravam-se 22 (9,4\%) dos animais. E entre os gatos que apresentavam idade superior a nove anos, estavam 23 (21,7\%) dos gatos.

A maior parte dos animais avaliados, 92 dos 106 (86,8\%), era sem raça definida, enquanto apenas 14 (13,2\%) eram de raça, sendo oito entre 14 gatos (57,1\%) eram siameses, três de 14 (21,4\%) eram persas e um destes 14 (7,1\%) pertenciam a cada uma das raças British Short Hair, Maine Coon e Sagrado da Birmânia, isto é, do total de 106 animais, 7,5\% eram siameses, 2,8\%, persas e 0,9\% cada uma das outras três raças.

\section{Dieta}

Dos 106 gatos, dois gatos não tiveram este item informado. Nenhum dos 104 animais se alimentava apenas uma vez ao dia. Gatos que se alimentavam duas vezes ao dia estavam em $15(14,2 \%)$ animais. Aqueles que se alimentavam três vezes ao dia estavam representados em 16 (15,4\%); os que comiam quatro vezes ao dia eram seis $(5,8 \%)$ e os gatos que se alimentavam cinco ou mais vezes ao dia foram identificados $67(64,4 \%)$ e apresentavam maior freqüência.

Trinta e três (15,38\%) gatos recebiam alimentos diferentes da dieta principal e 72 (68,57\%) felinos não se alimentavam com alimentos diferentes da sua dieta principal. Destes, $20(58,8 \%)$ animais tinham acesso a petiscos próprios para gatos, a peixe cozido 13 (38,2\%), a carne vermelha 11(32,4\%),

Tabela 1. Percentual de gatos ( $n=34)$ alimentados com diferentes tipos de alimentos diferente da alimentação principal.

\begin{tabular}{|c|c|c|}
\hline Alimento & $\begin{array}{l}\mathrm{n}^{\circ} \text { gatos que consomem } \\
\text { petisco }(\mathrm{n}=34)\end{array}$ & (\%) \\
\hline Próprio para gatos & 20 & 58,8 \\
\hline Peixe cozido & 13 & 38,2 \\
\hline Carne vermelha crua & 11 & 32,4 \\
\hline Frango cozido & 7 & 20,6 \\
\hline Atum enlatado & 7 & 20,6 \\
\hline Iogurte & 7 & 20,6 \\
\hline Frango cru & 6 & 17,6 \\
\hline Sardinha enlatada & 6 & 17,6 \\
\hline Peixe cru & 4 & 11,8 \\
\hline Presunto & 4 & 11,8 \\
\hline Carne vermelha cozida & 3 & 8,8 \\
\hline Peito de peru & 3 & 8,8 \\
\hline Ração seca & 2 & 5,9 \\
\hline Ração úmida & 2 & 5,9 \\
\hline Queijo & 2 & 5,9 \\
\hline Frutas & 1 & 2,9 \\
\hline Azeitona & 1 & 2,9 \\
\hline Biscoito salgado & 1 & 2,9 \\
\hline Mortadela & 1 & 2,9 \\
\hline Leite condensado & 1 & 2,9 \\
\hline Total & $102^{*}$ & -- \\
\hline
\end{tabular}

*Total referente aos gatos que consomem o petisco citado. Eventualmente os petiscos citados foram consumidos pelo animal. 
seguido de frango cozido, atum enlatado e iogurte com sete (20,6\%) animais cada. Frango cru e sardinha enlatada com 17 (17,6\%) gatos, peixe cru e presunto cru com quatro (11,6\%) cada, carne vermelha cozida e peito de peru com três (8,8\%) gatos, ração seca diferente da principal, ração úmida diferente da principal e queijo apresentaram dois $(5,9 \%)$ felinos e em menor freqüência estavam frutas, azeitona, biscoito salgado, mortadela e leite condensado com um único $(2,9 \%)$ animal cada (Tabela 1).

\section{Atividade física}

Cento e cinco respostas foram informadas. Trinta e dois (30,5\%) gatos faziam atividade física uma a duas vezes ao dia, nove (8,6\%) faziam atividade física três a quatro vezes ao dia, 26 (24,8\%) animais faziam atividade física mais que quatro vezes ao dia e seis (5,7\%) faziam exercício de forma esporádica. Trinta e dois (30,5\%) gatos não faziam atividade física. A área externa da residência era visitada por sete (6,7\%) dos gatos, enquanto, 98 (93,3\%) dos animais não tinham esse acesso.

\section{Perfil dos proprietários}

Um total de 50 proprietários assinalaram essa pergunta. Desses, 41 (82\%) eram do sexo feminino e 9 (18\%) do sexo masculino. A média de idade encontrada dos 50 proprietários foi de 29,36 $\pm 22,95$ anos. Dezenove (38\%) proprietários optaram por não divulgar a idade. Destes, 13 eram mulheres e cinco eram homens. Nenhum proprietário apresentou idade inferior a 23 anos e a maior idade foi 81 anos.

Foram observados 45/50 solteiros, sendo 16/45 mulheres e um homem; 16 casados, sendo 12/16 mulheres e quatro em 12 homens; em união estável seis pessoas, sendo quatro de seis eram mulheres e dois de seis eram homens; cinco pessoas separadas, sendo três mulheres e dois homens; divorciados, três mulheres e nenhum homem; e somente três mulheres eram viúvas e nenhum homem.

Com respeito ao tipo de moradia, dos 50 proprietários, observou-se que oito (16\%) proprietários moravam em casa; 40 (80\%) moravam em apartamento; eapenas dois (4\%) proprietários moravam em sobrado. Não foram encontrados proprietários morando em outro tipo de residência.

Com relação ao número de pessoas na residência, incluindo adultos, crianças e idosos, 50 residências foram avaliadas e obtiveram-se os seguintes dados: $22 / 50$ (44\%) residências com dois adultos, 10/50 (20\%) residências com 3 adultos, 6/50 (12\%) residências com quatro adultos, 3/50 (6\%) residências com cinco moradores e 1/50 (2\%) residências com oito adultos.

A grande maioria dos animais residia no município do Rio de Janeiro 45/50 (90\%), e apenas cinco (10\%) em outros municípios. Dos 45 proprietários que procediam do município do Rio de Janeiro, 11 eram da Tijuca (24,4\%), nove de Copacabana (20\%), seis de Botafogo (13,3\%), dois de Laranjeiras, Barra da Tijuca e Méier (4,5\%) cada. Com menor freqüência, Gávea, Jacarepaguá, Grajaú, Pilares, Ilha do Governador, Humaitá e Leblon com apenas um proprietário (2,2\%) cada. Dos cinco proprietários que não residiam no município do Rio de Janeiro dois eram de Niterói (40\%) e com menor freqüência de São Gonçalo, de Seropédica e de Édson Passos, todos com um (20\%) proprietário cada.

No que diz respeito à satisfação quanto à aparência do gato, 97/106 (91,5\%) gatos tiveram sua aparência apreciada por seus proprietários contra 9/106 (8,5\%) animais com seus proprietários insatisfeitos a aparência. Dos animais com a aparência apreciada 45/97 (46,4\%) estavam com peso corporal adequado e 52/97 (53,3\%) estavam com o peso inadequado. Já entre os gatos de proprietários insatisfeitos, foi notado que 4/9 (44,4\%) estavam com peso adequado e 5/9 (55,6\%) fora do peso ideal.

\section{O peso corporal e a classificação do sobrepeso}

O peso médio dos gatos foi de $4,80 \mathrm{~kg} \pm 1,98$, variando entre um mínimo de dois e um máximo de 12,1 kg. Dos gatos avaliados, a média do peso dos machos foi 5,35 $\pm 1,97$ e das fêmeas foi de $4,32 \pm 1,82$. A média do peso dos machos inteiros foi 3,84 $\pm 1,28$, a média dos machos castrados foi

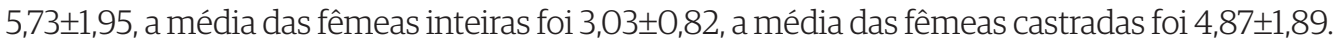

A escala de escore corporal variou entre 1 (caquético) e 5 (obeso) para os 106 gatos. Apenas dois (1,9\%) animais foram avaliados como caquéticos (escore 1). Na categoria magro (escore 2) foram apreciados nove gatos (8,5\%), e como ideal (escore 3) 29,2\% dos animais, ou seja, 31 gatos. 
Totalizando, então, 39,6\% (42/106) de gatos sem excesso de peso. O excesso de peso foi observado em 60,4\% (64/106); divididos nas categorias sobrepeso (escore 4), na qual foram encontrados 25 animais (23,6\%) e obeso (escore 5), na qual encontravam-se a maioria, 39 animais, isto é, 36,8\%.

Os animais avaliados com IMCF igual ou acima de $30 \%$ totalizaram 50 gatos, conferindo $47,2 \%$. Os felinos com IMCF entre 10 e menor 30\% são considerados dentro do peso ideal, esses totalizaram 54 (51\%) dos animais. Notaram-se dois animais com IMCF abaixo de 10\%, representando 1,9\% do total. Verificaram-se que, entre os animais de escore 5, 36/39 (92,3\%) tiveram IMCF calculado acima de 30\%. Já entre os animais com escore corporal 4, 14/25 (56\%) estavam com IMCF acima de $30 \%$, totalizando 50 animais com este valor de massa corporal, não tendo sido encontrados gatos com escore 1, 2 ou 3 nessa categoria (Figura 1).

\section{Análise dos fatores de risco}

Relativamente ao peso dos animais, observa-se correlação moderada altamente significativa ( $\mathrm{p}<0,05)$ entre proprietários e veterinários (coeficiente de correlação de Pearson: $\mathrm{r}=0,441 ; \mathrm{p}<0,0001$ ).

Dos 106 gatos avaliados, segundo a visão do veterinário 2/106 (50\%) animais foram considerados caquéticos, destes, o proprietário de um dos animais concordou com o escore do médico veterinário e o outro discordou, considerando seu animal com escore 3 (ideal). Para o escore 2 (magro) houve ocorrência de 9/106. Desses animais, 1/9 (11,11\%) foi considerado pelo proprietário caquético (escore 1), três foram considerados magros (escore 2) por seus proprietários e 5/9 animais foram considerados com escore ideal.

O escore ideal teve 31/106 animais. Destes, 4/31(12\%) animais foram considerados por seus proprietários com condição corporal caquética (escore 1), 4/31(12\%) atribuíram a condição magra (escore 2) para seus gatos, 17/31(57\%) gatos foram considerados dentro do peso ideal (escore 3), 6/31(19\%) animais foram considerados por seus proprietários com peso ideal (escore 4) por seus proprietários e nenhum destes foi classificado por seus proprietário como obeso (escore 5).

O sobrepeso foi identificado pelo médico veterinário em 25/106 animais, nenhum foi considerado caquético (escore 1), 1/25(4\%) animais foi considerado magro (escore 2) pelo proprietário, 9/25(36\%) animais foram considerados no escore ideal (escore 3) pelo proprietário, 15/25 (36\%) animais foram considerados como com sobrepeso (escore 4) pelo proprietário e nenhum proprietário classificou seu gato como obeso.

O escore obeso (escore 5) foi identificado pelo médico veterinário em 39/106 animais, nenhum foi considerado caquético (escore 1), 1/39(2,6\%) animal foi julgado magro (escore 2) por seu proprietário, 8/39 (20,5\%) foram considerados como ideal (escore 3) pelo proprietário, 25/39(64,1\%) foram classificados com sobrepeso (escore 4) e 5/39 (64,1\%) indicados como obesos (escore 5) por seus proprietários. Houve uma fraca concordância, embora altamente significativa ( $\mathrm{p}<0,01)$, entre a estimativa ECC indicado pelo proprietário com a avaliação do veterinário ( $\kappa=0,195 ; \mathrm{p}<0,0001)$.

\section{Fatores de risco relacionados aos métodos subjetivo e método objetivo de avaliação da obesidade}

O estado reprodutivo dos gatos tomou como referência a classe em maior ocorrência (fêmea inteira). Quando relacionado o estado reprodutivo com o ECC não foi observada associação para sobrepeso e obesidade (FC, OR=15,63 IC95\%: 3,58-68,20), (MC, OR=19,44 95\% IC:0,4,61-82,04),

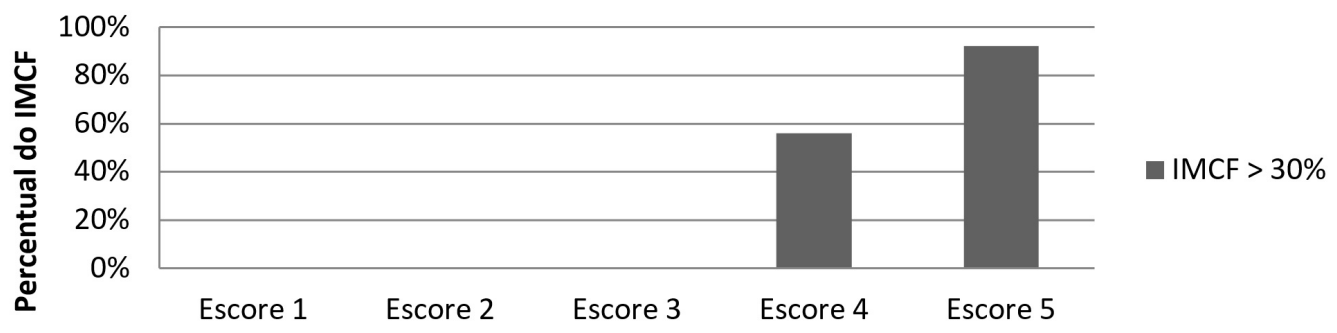

Figura 1. Distribuição (em porcentagem) dos gatos com índice de massa corporal felino maior que $30 \%$. Observa-se que todos apresentavam escore de sobrepeso ou obesidade, ou seja, 4 ou 5. Não houve ocorrência deste IMCF nos animais com escore 1, 2 ou 3. Rio de Janeiro, 2011. 
Tabela 2. Dados brutos e o intervalo de confiança de gatos com sobrepeso (ECC 1 a 3) e obesidade (ECC = 4 e 5) comparado aos gatos não obesos e sem sobrepeso (ECC=1 a 3).

\begin{tabular}{|c|c|c|c|c|c|}
\hline Variável & & (ECC 1 a 3) & ECC 4 a 5) & OR & $95 \% \mathrm{CI}$ \\
\hline \multirow{4}{*}{ Estado reprodutivo } & $\mathrm{FC}$ & 8 & 25 & 15,63 & $3,58-68,20$ \\
\hline & FI & 15 & 3 & 1,00 & - \\
\hline & $\mathrm{MC}$ & 9 & 35 & 19,44 & $4,61-82,04$ \\
\hline & MI & 10 & 1 & 0,5 & $0,05-5,51$ \\
\hline \multirow{6}{*}{ Raça } & SRD & 32 & 60 & 1,00 & - \\
\hline & Siamês & 5 & 3 & 0,32 & $0,07-1,43$ \\
\hline & Persa & 2 & 1 & 0,27 & $0,02-3.09$ \\
\hline & Sagrado da Birmânia & 1 & 0 & 0,18 & $0,01-4,55$ \\
\hline & Maine Coon & 1 & 0 & 0,18 & $0,01-4,55$ \\
\hline & British Short Hair & 1 & 0 & 0,18 & $0,01-4,55$ \\
\hline \multirow{5}{*}{ Faixa etária dos gatos } & $1 \mathrm{a} 2$ anos & 22 & 14 & 1,00 & - \\
\hline & $<$ lanos & 7 & 0 & 0,1 & $0,01-1,89$ \\
\hline & $>2$ a 5 anos & 5 & 5 & 1,57 & $0,38-6,43$ \\
\hline & $>5$ anos a 9 anos & 3 & 19 & 9,95 & $2,48-39,94$ \\
\hline & $>9$ anos & 5 & 18 & 5,66 & $1,71-18,72$ \\
\hline \multirow{2}{*}{ Acesso ao alimento } & Livre & 40 & 48 & 1,00 & - \\
\hline & Controlado & 1 & 16 & 13,33 & $1,69-104,95$ \\
\hline \multirow{5}{*}{ Freqüência da alimentação } & $1 \times$ dia & 0 & 0 & 0,53 & $0,01-27,57$ \\
\hline & $2 \mathrm{x}$ dia & 6 & 9 & 0,78 & $0,25-2,46$ \\
\hline & $3 \times$ dia & 10 & 6 & 0,31 & $0,01-0,96$ \\
\hline & $4 \times$ dia & 2 & 4 & 1,05 & $0,18-6,17$ \\
\hline & $5 \mathrm{x}$ ou mais & 23 & 44 & 1,00 & - \\
\hline \multirow{2}{*}{ Ingestão de petiscos } & Sim & 13 & 20 & 0,98 & $0,42-2,28$ \\
\hline & Não & 28 & 44 & 1,00 & - \\
\hline \multirow{2}{*}{ Atividade física } & Sim & 30 & 47 & 0,99 & $0,41-2,40$ \\
\hline & Não & 11 & 17 & 1,00 & - \\
\hline \multirow{4}{*}{ Freqüência da atividade física } & 1 a 2 vezes & 3 & 29 & 21,75 & $5,09-92,85$ \\
\hline & 2 a 4 vezes & 4 & 5 & 2,81 & $0,59-92,85$ \\
\hline & Mais que 4 vezes & 18 & 8 & 1,00 & - \\
\hline & Esporádico & 2 & 4 & 4,5 & $0,68-29,81$ \\
\hline \multirow{2}{*}{ Acesso à áreas externas } & Sim & 6 & 1 & 0,09 & $0,01-0,78$ \\
\hline & Não & 35 & 63 & 1,00 & - \\
\hline \multirow{2}{*}{ Tipo de alimentação } & Somente seca & 40 & 57 & 1,00 & - \\
\hline & Úmida e seca & 1 & 7 & 4,91 & $5,58-41,48$ \\
\hline \multirow{3}{*}{ Faixa etária do proprietário ( $n=27)$} & 20-40 anos & 16 & 27 & 1,00 & - \\
\hline & $41-65$ anos & 13 & 16 & 0,73 & $0,28-1,90$ \\
\hline & Acima de 66 & 0 & 6 & 7,8 & $0,41-147,62$ \\
\hline \multirow{4}{*}{ Número de crianças na residência } & Nenhuma & 28 & 53 & 1,00 & - \\
\hline & 1 & 13 & 6 & 0,24 & $0,08-0,70$ \\
\hline & 2 & 0 & 5 & 5,86 & $0,31-109,81$ \\
\hline & 3 ou mais & 1 & 0 & 0,18 & $0,01-4,56$ \\
\hline \multirow{2}{*}{ Escore subestimado pelo proprietário } & Não & 37 & 13 & 1,00 & - \\
\hline & Sim & 5 & 51 & 29,03 & $9,52-88,52$ \\
\hline \multirow{3}{*}{ Quem fornece o alimento } & Proprietário & 33 & 41 & 1,00 & - \\
\hline & Funcionário & 6 & 13 & 1,74 & $0,60-5,08$ \\
\hline & Parente & 3 & 14 & 3,76 & $1,00-14,20$ \\
\hline
\end{tabular}

ECC = escore de condição corporal, $\mathrm{FI}$ = fêmeas inteiras, $\mathrm{FC}$ = fêmeas castradas, $\mathrm{MI}$ = macho inteiro, $\mathrm{MC}$ = macho castrado, SRD = sem raça definida. 
Tabela 3. Número absoluto, odds ratio (OR) intervalo de confiança a 95\% (95\%IC) de gatos obesos (IMCF > 30\%) e não obesos (IMCF < 30\%).

\begin{tabular}{|c|c|c|c|c|c|}
\hline Variável & & IMCF $<30 \%$ & IMCF $\geq 30 \%$ & OR & 95\%IC \\
\hline \multirow{4}{*}{ Estado reprodutivo } & $\mathrm{FC}$ & 16 & 17 & 18,06 & $2,15-151,86$ \\
\hline & FI & 17 & 1 & 1,00 & - \\
\hline & $\mathrm{MC}$ & 13 & 31 & 40,54 & $4,87-337,16$ \\
\hline & MI & 1 & 10 & 170,00 & $9,54-3027,87$ \\
\hline \multirow{6}{*}{ Raça } & SRD & 44 & 48 & 1,00 & - \\
\hline & Siamês & 5 & 3 & 0,55 & $0,01-2,44$ \\
\hline & Persa & 3 & 0 & 0,13 & $0,02-2,59$ \\
\hline & Sagrado da Birmânia & 1 & 0 & 0,92 & $0,02-47,36$ \\
\hline & Maine coon & 1 & 0 & 0,92 & $0,02-47,36$ \\
\hline & Brithsh short hair & 1 & 0 & 0,92 & $0,02-47,36$ \\
\hline \multirow{5}{*}{ Faixa etária dos gatos } & $1 \mathrm{a} 2$ anos & 27 & 9 & 1,00 & - \\
\hline & $<$ lanos & 7 & 0 & 0,19 & $0,01-3,65$ \\
\hline & $>2$ a 5 anos & 7 & 3 & 1,29 & $0,27-6,07$ \\
\hline & $>5$ anos a 9 anos & 5 & 17 & 10,20 & $2,92-35,61$ \\
\hline & $>9$ anos & 7 & 16 & 6,86 & $2,14-22,00$ \\
\hline \multirow{2}{*}{ Acesso ao alimento } & Livre & 52 & 36 & 1,00 & - \\
\hline & Controlado & 3 & 14 & 6,74 & $1,81-25,16$ \\
\hline \multirow{5}{*}{ Freqüência da alimentação } & $1 \mathrm{x}$ dia & 0 & 0 & 0,86 & $0,02-44,61$ \\
\hline & $2 \mathrm{x}$ dia & 9 & 6 & 0,57 & $0,18-1,78$ \\
\hline & $3 x$ dia & 12 & 4 & 0,29 & 0,08-0,99 \\
\hline & $4 \times$ dia & 3 & 3 & 0,86 & $0,16-4,57$ \\
\hline & 5 ou mais vezes & 31 & 36 & 1,00 & - \\
\hline \multirow{2}{*}{ Ingestão de petiscos } & Sim & 18 & 15 & 0,93 & $0,41-2,13$ \\
\hline & Não & 38 & 34 & 1,00 & - \\
\hline \multirow{2}{*}{ Atividade física } & Sim & 38 & 39 & 1,00 & - \\
\hline & Não & 18 & 10 & 0,54 & $0,22-1,32$ \\
\hline \multirow{4}{*}{ Frequência da atividade física } & 1a 2 vezes & 7 & 25 & 27,38 & $6,32-118,64$ \\
\hline & 3 a 4 vezes & 4 & 5 & 9,58 & $1,61-56,93$ \\
\hline & Mais que 4 vezes & 23 & 3 & 1,00 & - \\
\hline & Esporádico & 3 & 3 & 7,67 & $1,04-77,43$ \\
\hline \multirow{2}{*}{ Acesso à áreas externas } & Sim & 6 & 1 & 0,17 & $0,02-1,46$ \\
\hline & Não & 49 & 49 & 1,00 & - \\
\hline \multirow{2}{*}{ Tipo de alimentação } & Somente seca & 55 & 42 & 1,00 & - \\
\hline & Úmida e seca & 1 & 7 & 9,17 & $1,09-77,43$ \\
\hline \multirow{3}{*}{ Faixa etária do proprietário $(n=27)$} & 20-40 anos & 24 & 19 & 1,00 & \\
\hline & 41-65 anos & 16 & 13 & 1,03 & $0,40-2,66$ \\
\hline & Acima de 66 & & 6 & 16,33 & 0,87-308,08 \\
\hline \multirow{4}{*}{ Número de crianças na residência } & Nenhuma & 39 & 48 & 1,00 & - \\
\hline & 1 & 16 & 3 & 0,15 & $0,04-0,55$ \\
\hline & 2 & 0 & 5 & 8,96 & $0,48-167,03$ \\
\hline & 3 ou mais & 1 & 0 & 0,27 & $0,01-6,81$ \\
\hline \multirow{2}{*}{ Escore subestimado pelo proprietário } & Não & 42 & 8 & 1,00 & - \\
\hline & Sim & 14 & 42 & 15,75 & $1,12-11,03$ \\
\hline \multirow{3}{*}{ Quem fornece o alimento } & Proprietário & 44 & 30 & 1,00 & - \\
\hline & Funcionário & 7 & 12 & 2,51 & 0,89-7,11 \\
\hline & Parente & 5 & 12 & 3,52 & 1,12-11,03 \\
\hline
\end{tabular}

IMCF = índice de massa corporal do felino, FI = fêmeas inteiras, FC = fêmeas castradas, $\mathrm{MI}$ = macho inteiro, MC = macho castrado, SRD = sem raça definida. 
(MI, OR=15,6 IC95\%: 0,05-5,51) (Tabela 2). Quanto ao estado reprodutivo dos gatos relacionado ao IMCF, comparando com fêmeas inteiras as demais classes, observamos que a castração para fêmeas e machos é um fator de risco para obesidade (FC, OR=18,06 IC95\%: 2,15-151,86), (MC, OR=40,54 95\%IC:4,87-337,16), (MI, OR=170,00 IC95\%:9,54-3027,87) (Tabela 3). Para o ECC e IMCF, não houve associação das raças encontradas com a obesidade neste estudo (Tabela 3).

Tomando por referência a faixa de idade mais numerosa (de um a dois anos), a associação significativa da idade com o ECC mostrou que o risco de sobrepeso foi dez vezes maior (OR=9,95; IC95\%: 2,48 a 39,94) nos gatos entre cinco e nove anos. Esse risco para os gatos com mais de nove anos foi significativo e alcançou cerca de cinco vezes e meia a mais do que o dos gatos da faixa de um a dois anos (OR=5,66; IC95\%: 1,71 a 18,72). As demais faixas etárias não apresentaram associação significativa com o ECC (Tabela 2). A correlação do IMCF com a idade, tomando como referência a faixa etária mais numerosa (de um a dois anos), mostrou-se semelhante à associação com o ECC: o risco de sobrepeso foi dez vezes maior (OR=10,20; IC 95\%: 2,92 a 35,61) nos gatos com mais de cinco e até nove anos. Esse risco para os gatos com mais de nove anos foi significativo e alcançou cerca de sete vezes a mais do que o dos gatos da faixa de um a dois anos (OR=6,86; IC95\%: 2,14 a 22,00). As demais faixas etárias não apresentaram associação significativa com o IMCF (Tabela 3).

Oacesso livre ao alimento quando comparado ao ECC éum fator de risco (95\% IC 1,69-104,95) (Tabela 2), assim como quando comparamos o IMCF ao acesso livre ou controlado ao alimento (95\% IC 1,81-25,16) (Tabela 3). A freqüência da alimentação relacionada ao escore e ao IMCF mostrou um fator de proteção (OR=0,31; IC 95\%: 0,01-0,96) (Tabela 2) e (OR=0,29 95\% IC 0,08-0,99) (Tabela 3), respectivamente, para os gatos que comem três vezes ao dia quando comparados aos que comem cinco ou mais vezes ao dia. Quando foi correlacionado o tipo de alimentação com ECC e o com IMCF dos gatos que comem rações úmidas e secas encontrou-se um fator de risco cerca de quatro vezes maior (OR=4,91, IC95\%: 5,58-41,48) (Tabela 2) e nove vezes maior (95\% IC 1,09-77,43), (Tabela 3), respectivamente, de desenvolver o sobrepeso e obesidade quando comparados aos que comem somente alimento seco.

Com relação ao consumo de alimentos diferentes da alimentação principal (petiscos), não houve associação significativa entre os gatos que comem e os que não comem tanto na avaliação do escore como para IMCF.

Já com relação à atividade física e o ECC observamos um fator de risco para gatos que fazem atividade física na freqüência de uma a duas vezes por dia (OR=21,75, IC 95\%: 5,09-92,85) (Tabela 2). Já para a correlação do IMCF com a atividade física observamos um fator de risco para todas as variáveis de gatos que fazem atividade física mais que quatro vezes ( 1 a 2 vezes OR=27,38, IC95\%: 6,32-118,64; 3 a 4 vezes OR= 9,58, IC 95\%: 1,61-56,93 e esporádico OR=7,67, IC 95\%: 1,04-77,43) (Tabela 3).

O acesso a áreas externas da casa quando correlacionado ao ECC não mostrou associação para obesidade (Tabela 2), enquanto quando relacionado ao IMCF foi um fator de proteção (OR=0,09, IC95\%: 0,01-0,78) (Tabela 3).

Com relação à subestimação do ECC quando avaliado pelo proprietário e confrontado com o ECC do médico veterinário o risco é de 29 vezes maior (OR=29,03, IC95\%: 9,52-88,52) (Tabela 2) para a obesidade e o sobrepeso. Do ponto de vista do IMCF, a subestimação da obesidade do gato pelo proprietário eleva em 15 vezes (OR=15,75, IC 95\%: 1,12-11,03) (Tabela 3) a chance desse animal desenvolver obesidade.

\section{Presença das doenças relacionadas com a obesidade}

Para avaliar as doenças relacionadas à obesidade, os animais foram distribuídos em dois grupos com base no IMCF. Grupo 1: gatos não obesos. Composto por 56/106 gatos. Grupo 2: gatos obesos. Com 50/106 gatos. Assim, obtivemos a seguinte distribuição: grupo 1, 4/56 (7,1\%, IC 95\%: 7,0---7,2\%) apresentavam doença do trato urinário inferior, 3/56 (5,4\%, IC 95\%: 5,3---5,5\%) doenças da cavidade oral, 2/56 (3,6\%, IC 95\%: 3,6---3,6\%) apresentavam dermatopatias e gastropatias. No grupo 2 obtivemos a seguinte distribuição: 11/50 (22\%, IC 95\%: 21,9---22,1\%) apresentavam doença da cavidade oral. Diabete, dermatopatias e neoplasias apresentaram 4/50 (8\%, IC 95\%: 7,9---8,1\%) cada, 2/50 (4\%, IC 95\%: 3,9---4,1\%) gastropatias e, por último, a doença do trato urinário inferior com 2/50 gatos (4\%, IC 95\%: 3,9---4,1\%). Das doenças não 
relacionadas diretamente à obesidade obteve-se a seguinte freqüência: 14/50 (28\%, IC 95\%: 13,1--13,3\%) hipertireoidismo, 3/50 (6\%, IC 95\%: 5,9---6,1\%) insuficiência renal crônica e 1/50 (2\%, IC 95\%: 0,9---0,9\%) neuropatia.

\section{Discussão}

Este é o primeiro estudo realizado na região metropolitana do Rio de Janeiro avaliando a condição corporal de gatos, por meio de uma análise subjetiva e objetiva e os possíveis fatores de riscos associados à obesidade felina.

Não se pode afirmar que a população do estudo representa a população total de gatos do Rio de Janeiro, por isso deve-se analisar com cautela, uma vez que o estudo foi realizado numa clínica veterinária voltada somente para o atendimento de felinos domésticos e com animais da rotina cirúrgica, mostrando apenas o perfil desta realidade. Este fato pode ocultar ou mesmo elevar a freqüência de um determinado perfil de gatos. Todavia, os gatos são oriundos de treze bairros e cinco municípios distintos e nossa população de animais apresenta um número razoável ( $\mathrm{n}=06$ ).

Neste estudo, o excesso de peso foi identificado na metade dos animais que se submeteram a algum tipo de intervenção cirúrgica, o que deve ser considerado como um alerta para o proprietário de gatos, visto que o risco anestésico é maior nesta condição (Lund et al., 2005).

A idade destes gatos se mostrou um fator menor de risco para os gatos jovens. Jáa chance dos gatos se tornarem obesos foi dez vezes maior na faixa etária entre cinco e nove anos, e de seis vezes maior para animais com mais de nove anos corroborando com outros autores (Lund et al., 2005; Colliard et al., 2009; Courcier et al., 2010). Notamos também que gatos com idade mais avançada (acima de nove anos) têm uma redução da massa corporal (Lund et al., 2005).

Uma predisposição racial significante à obesidade tem sido demonstrada (Byers et al., 2011) . O grupo preponderante dos gatos foi de animais mestiços dificultando a associação com a obesidade, embora a quase totalidade dos gatos obesos não tivessem raça defina.

No presente estudo o risco para obesidade foi maior tanto para fêmeas quanto para machos castrados (Scarlett \& Donoghue, 1998; Allan et al., 2000; Russell et al., 2000; Martin et al., 2006; Lund et al., 2005). A esterilização resulta na diminuição dos hormônios sexuais, que afetam diretamente o centro da saciedade no sistema nervoso central por meio de alterações nas concentrações de leptina e grelina. Além do que, é possível que ocorram, de forma indireta, alterações no metabolismo celular e nos reguladores hormonais do alimento (Byers et al., 2011)

A chance destes gatos adquirirem sobrepeso e até se tornarem obesos foi de 13 e seis vezes, maior, respectivamente, para os felinos que têm acesso livre à comida. Estes buscam, provavelmente, uma complementação alimentar (Allan et al., 2000; Kienzle \& Bergler, 2006). A maior parte dos animais também era alimentada mais que cinco vezes, ou na forma ad libitum confirmando algumas publicações (Scarlett \& Donoghue, 1998; Allan et al., 2000; Russell et al., 2000; Courcier et al., 2010) e mostrou-se diferente de outros autores (Robertson, 1999; Lund et al., 2005; Colliard et al., 2009).

A dieta foi composta basicamente de ração seca ou úmida associada à seca. Nenhum dos animais consumia restos de comida, comida caseira ou somente ração úmida. Acredita-se que isto demonstrou a preferência dos proprietários em ofertar alimentos secos balanceados para gatos e, possivelmente, não pesam e não mensuram a quantidade certa de ração a ser oferecida para seus animais, como relatam outros estudos (Robertson, 1999; Colliard et al., 2009). Assim, é válido ressaltar que a dieta não deve ser prescrita pelo médico veterinário identificando apenas um nome ou "tipo" de alimento. Ele deve também indicar a quantidade e a freqüência que a ração deve ser oferecida ao gato.

O consumo de petiscos não foi um fator de risco nesta pesquisa, isso pode ter ocorrido, talvez, por dois motivos: 1) receio do proprietário de informar este item,2) por esses felinos ficarem a maior parte do tempo com outro morador e, possivelmente, este ser o responsável pelo fornecimento dos petiscos. Entre os petiscos oferecidos aos gatos, metade era do tipo próprios para gatos, seguido de peixe cozido, carne crua, frango cozido, iogurte e atum enlatado. Ainda não há um consenso ou explicação consistente sobre efeito da alimentação e introdução de petiscos com a obesidade em gatos (Courcier et al., 2010), sendo assim, mais estudos devem ser direcionados a este tópico.

A falta de atividade física tem sido apontada como um fator predisponente para obesidade (Courcier et al., 2010), fato não observado nesta avaliação. Não obstante, os gatos que não praticam 
atividade física, independente da freqüência, tinham quase 30\% mais chance de adquirir obesidade. Além disso, a chance de ter sobrepeso reduziu conforme o aumento da freqüência da atividade.

Nossos gatos domiciliados apresentaram maior freqüência para obesidade, mostrando que vida livre pode ser um fator protetor contra o excesso de peso corroborando com Allan et al. (2000). Essas diferenças podem estar na formulação dos questionários. Está claro também que a obesidade não é simplesmente um caso de ingestão calórica excessiva ou "gula" uma vez que fatores não-alimentares, tais como comportamento e estilo de vida podem contribuir para o desenvolvimento dessa condição.

No que diz respeito aos proprietários, quase todos eram do sexo feminino e residiam em apartamento. Encontramos um fator protetor para a obesidade em residências onde mora só uma criança, possivelmente por seus proprietários focarem seu zelo para a criança, reduzindo a atenção com a dieta do gato. $\mathrm{O}$ fornecimento do alimento por um parente do dono do gato mostrou-se um discreto fator de risco, tendo três vezes mais chance de causar obesidade. Possivelmente, os gatos alimentados por "terceiros" poderiam estar recebendo alimento a mais ou de forma errada, pois esta pessoa não teria ciência da quantidade e/ou da freqüência com que deveria ser administrada a ração ou ainda não compreender a importância deste item para qualidade de vida do gato.

O peso médio dos gatos foi de 4,8 kg. A média do peso dos machos foi superior as fêmeas, mesmo quando esses eram castrados (Lund et al., 2005). A média de peso dos animais castrados também foi superior aos não castrados como já demonstrado por outros autores (Lund et al., 2005; Colliard et al., 2009; Courcier et al., 2010).

Os dois tipos de avaliações de obesidade foram efetivas em demonstrar que cerca de metade dos gatos apresentaram excesso de peso. O método objetivo (IMCF) mostrou-se pouco mais preciso no que diz respeito à obesidade quando comparado à graduação do escore, visto que metade dos gatos foi graduado com escore 4 e estes já apresentavam percentuais acima de 30\%. O contrário também ocorreu, embora em menor proporção. O IMCF mostrou-se um método mais preciso para a confirmação da obesidade e deve ser empregado para conscientizar o proprietário acerca da condição de seu animal. $\mathrm{O}$ índice de massa corporal pode ser o primeiro degrau para distinguir a obesidade felina propriamente dita. Este tipo de avaliação pode ser uma ferramenta especialmente útil e precisa para convencer os proprietários de que seu gato está com excesso de peso e deve emagrecer.

A graduação da condição corporal é uma maneira bem simples, embora necessite de treino do observador. Donos devem, também, ser "treinados" a avaliar seus animais frequentemente, pois é a forma mais "precoce" de identificar o excesso de peso. Tanto a forma de avaliação por escore quanto o cálculo da massa corporal devem fazer parte da rotina clínica dos gatos de forma a prevenir o desenvolvimento de sobrepeso. Essas avaliações também são importantes para visualizar uma perda de peso, pois este pode ser o primeiro sinal de uma doença. É provável que a associação desses dois métodos possa proporcionar um diagnóstico melhor da condição corporal e ajudar o veterinário a traçar estratégias adequadas para o tratamento.

No que diz respeito à satisfação com a aparência do gato, a maioria absoluta dos proprietários estava satisfeita e pouco mais da metade destes animais apresentou peso inadequado. Já os gatos que tinham proprietários insatisfeitos com a aparência, metade estava com peso adequado. Isso evidencia o fato de que os animais estarem acima do peso, não incomoda seus donos, que ficam satisfeitos com a aparência mais "gordinha" de seus gatos, não correlacionando com possíveis enfermidades a curto e longo prazo.

A comparação entre as percepções do proprietário e a do médico veterinário mostrou que quanto menor o escore, mais próxima está a percepção do proprietário à do médico veterinário. Uma discreta superestimação do escore pelo dono o também ocorreu. Conforme a graduação da condição corporal aumentava, maior era a diferença entre o escore do médico veterinário e do proprietário. Quando o escore era três, notamos uma proporção semelhante entre a subestimação e a superestimação pelo proprietário, mas a maioria concordava com o escore do médico veterinário. À medida que a condição corporal elevava, observou-se que a subestimação também aumentava, chegando a ser a maioria para os gatos obesos. O presente trabalho mostrou que esta subestimação tinha nove vezes mais chance de ocorrer, quando usada a avaliação objetiva e 30 vezes mais com a avaliação subjetiva. Estes resultados podem indicar que os donos desses 
gatos não conseguem distinguir o que é excesso de peso ou mesmo obesidade para os felinos. Assim, reforçamos que a conscientização do proprietário é o primeiro passo para a prevenção desta doença (Allan et al., 2000; Colliard et al., 2009).

A associação de doenças documentadas aqui é de especial interesse já que fornece evidência adicional da relação entre excesso de peso ou obesidade e doenças específicas nos gatos. Nesta amostra, os gatos obesos apresentavam maior incidência de doenças bucais, diabetes, gastropatias e neoplasias que o resto da população como relatado por Lund et al. (2005). Gatos obesos têm um risco aumentado para a doença do trato urinário (Lund et al., 2005), embora, em nosso estudo, a freqüência desta doença tenha sido maior em gatos não obesos. Doenças ligadas à cavidade oral mostraram-se as mais freqüentes entre as afecções relacionadas à obesidade em gatos, diferente de outros autores que indicam que o diabetes é a doença mais comumente encontrada em felinos obesos (Scarlett \& Donoghue, 1998; Lund et al., 2005).

Um dado que surpreendeu foi a elevada freqüência do hipertireoidismo em nossa amostra, na qual obtivemos cerca 30\% de gatos obesos com esta afecção. Esta endocrinopatia é das mais comuns em felinos adultos e é caracterizada por inúmeras alterações no metabolismo de vários sistemas e tem como um dos sinais clínicos mais comuns o emagrecimento. Gatos obesos podem apresentar esta endocrinopatia, fato este encontrado num estudo em gatos com hipertireoidismo no Rio de Janeiro (Farias, 2009), portanto, esta doença deve ser pesquisada precocemente em gatos adultos obesos. A doença renal também foi observada em gatos obesos, porém em baixa freqüência. Estas afecções têm influência sobre a condição corporal dos gatos e pode variar de acordo com o grau de catabolismo ou anabolismo destes animais, daí mais uma razão, para inclusão destas avaliações na rotina clínica de todos os gatos independente da idade ou mesmo da presença de doenças.

Além de fornecer dados descritivos sobre a obesidade em gatos no Rio de Janeiro, os fatores relatados neste estudo podem ajudar aos médicos veterinários a avaliarem quais felinos possuem predisposição ao excesso de peso ou mesmo à obesidade. A prevenção da obesidade felina pode ser tão ou mais importante que sua cura.

\section{Conclusões}

A freqüência de excesso de peso na população de gatos da rotina cirúrgica foi de $60,4 \%$, sendo 23,6\% identificados com sobrepeso e 36,8\% obesos através da avaliação do escore corporal.

A obesidade foi evidenciada em metade desta população estudada pelo método do índice de massa corporal do felino. Os fatores de risco para o excesso de peso foram gatos castrados, especialmente machos, de meia idade (entre cinco e nove anos), domiciliados e com acesso livre ao alimento.

A subestimação da condição corporal dos gatos pelos proprietários foi um fator predisponente para obesidade felina.

A associação dos métodos subjetivo (ECC) com o objetivo (IMCF) são formas determinantes e concisas para uma adequada avaliação da obesidade.

\section{Referências}

Allan, F. J., Pfeiffer, D. U., Jones, B. R., Esslemont, D. H. B., \& Wiseman, M. S. (2000). A cross-sectional study of risk factors for obesity in cats in New Zealand. Preventive Veterinary Medicine, 46(3), 183-196. http://dx.doi. org/10.1016/S0167-5877(00)00147-1. PMid:10913803.

Butterwick, R. F., \& Markwell, P. J. (1996). Changes in the body composition of cats during weight reduction by controlled dietary energy restriction. The Veterinary Record, 138(15), 354-357.

Byers, C. G., Netting, F. E., Olsen, C., Stephens, M. B., Wilson, C. C., \& Goodie, J. (2011). Exploring the causes and consequence of canine obesity. Veterinary Medicine, 1, 184-190.

Colliard, L., Paragon, B., Lemuet, B., Bénet, J. J., \& Blanchard, G. (2009). Prevalence and risk factors of obesity in an urban population of healthy cats. Journal of Feline Medicine and Surgery, 11(2), 135-140. http://dx.doi. org/10.1016/j.jfms.2008.07.002. PMid:18774325.

Courcier, E. A., O'Higgins, R., Mellor, D. J., \& Yam, P. S. (2010). Prevalence and risk factors for feline obesity in a first opinion practice in Glasgow, Scotland. Journal of Feline Medicine and Surgery, 12(10), 746-753. PMid:20685143.

Crystal, M. A. (2009). Obesity. In G. D. Norsworthy, M. A. Crystal, S. F. Grace, \& L. P. Tilley (Eds.), O paciente felino (3rd ed., pp. 223-224). São Paulo: Roca. 
Farias, V. P. (2009). Avaliação clínica, laboratorial e ecocardiográfica de gatos domésticos hipertireóideos no período entre 2007 e 2008 (Dissertação de mestrado). Universidade Federal Rural do Rio de Janeiro, Seropédica.

German, A. J. (2006). The growing problem of obesity in dogs and cats. The Journal of Nutrition, 136(7, Suppl), 1940S-1946S. http://dx.doi.org/10.1093/jn/136.7.1940S. PMid:16772464.

German, A. J., \& Martin, L. (2009). Feline obesity: epidemiology, pathophysiology and management. In P. Pibot, V. Biourge \& D. Elliot (Eds.), Encyclopedia of feline nutrition. Aimargues, France: Aniwa SAS. Recuperado em 8 de março de 2010, de http://www.ivis.org/advances/rcfeline/toc.asp

Kienzle, E., \& Bergler, R. (2006). Human-animal relationship of owners of normal and overweight cats. The Journal of Nutrition, 136(7, Suppl), 1947S-1950S. http://dx.doi.org/10.1093/jn/136.7.19475. PMid:16772465.

Laflamme, D. P. (1997). Development and validation of a body condition score system for cats: a clinical tool. Feline Practice, 25, 13-18.

Laflamme, D. P. (2006). Understanding and managing obesity in dogs and cats. The Veterinary Clinics of North America. Small Animal Practice, 36(6), 1283-1295, vii. http://dx.doi.org/10.1016/j.cvsm.2006.08.005. PMid:17085235.

Lund, E. M., Armstrong, P. J., Kirk, C. A., \& Klausner, J. S. (2005). Prevalence and risk factors for obesity in adult cats from private us veterinary practices. International Journal of Applied Research in Veterinary Medicine, 3(2), 88-96.

Martin, L., Siliart, B., Dumon, H., \& Nguyen, P. (2006). Spontaneous hormonal variations in male cats following gonadectomy. Journal of Feline Medicine and Surgery, 8(5), 309-314. http://dx.doi.org/10.1016/j.jfms.2006.03.002. PMid:16713320.

Robertson, I. D. (1999). The influence of diet and other factors on owner- perceived obesity in privately owned cats from metropolitan Perth, Western Australia. Preventive Veterinary Medicine, 40(2), 75-85. http://dx.doi. org/10.1016/S0167-5877(99)00024-0. PMid:10384945.

Russell, K., Sabin, R., Holt, S., Bradley, R., \& Harper, E. J. (2000). Influence of feeding regimen on body condition in the cat. The Veterinary Clinics of North America. Small Animal Practice, 41(1), 12-17. PMid:10713977.

Scarlett, J. M., \& Donoghue, S. (1998). Association between body condition and disease in cats. Journal of the American Veterinary Medical Association, 212(11), 1725-1731. PMid:9621878.

Scarlett, J. M., Donoghue, S., Saidla, J., \& Wills, J. (1994). Overweight cats: prevalence and risk factors. International Journal of Obesity and Related Metabolic Disorders, 18(1, Suppl 1), S22-S28. PMid:8087161.

Zoran, D. L. (2010). Obesity in dogs and cats: a metabolic and endocrine disorder. The Veterinary Clinics of North America. Small Animal Practice, 40(2), 221-239. http://dx.doi.org/10.1016/j.cvsm.2009.10.009. PMid:20219485. 